\title{
Gated SPECT myocardial perfusion scintigraphy: a multi-faceted tool for the evaluation of heart failure
}

\author{
Prem Soman, MD, PhD, FRCP
}

\section{See related article, pp. 212-221}

Compared to the large volume of data available on the prognostic utility of myocardial perfusion scanning (MPS) in unselected patients referred for stress testing and several subgroups therein, there is only a relatively scant amount of literature pertaining specifically to the use of MPS to determine prognosis in patients with heart failure. One could be excused for being surprised at this state of affairs, since heart failure is widely prevalent, and frequently encountered by cardiologists, internists, and specialists in cardiac imaging, and MPS is one of the most widely utilized cardiac assessment tools.

In this issue of the Journal Candell-Riera and colleagues report on the prognostic value of clinically indicated Tc-99m gated SPECT MPS in a prospective cohort of patients with left ventricular ejection fraction (LVEF) $\leq 40 \%$. Of 6114 patients referred to the authors' laboratory for MPS over a 6-year period, 365 had LVEF $\leq 40 \%$. After excluding patients with nonischemic LV dysfunction and censuring patients who had early (with 60 days of MPS) revascularization, 167 patients with ischemic cardiomyopathy were followed up for an average of $2.3 \pm 1.2$ years to identify the variables associated with cardiac death. Thirty patients had stress testing precluded by severe heart failure and therefore, underwent only rest gated MPS. The mortality rate over this relatively short period of follow-up was substantial: $22 \%(36 / 167)$ all-cause mortality, of which $17 \%(29 /$ 167) were cardiac deaths. As expected, the majority of deaths in this population were from progressive heart failure (23), and most of the remaining were sudden cardiac deaths.

From the University of Pittsburgh Medical Center-Cardiovascular Institute, Pittsburgh, PA.

Reprint requests: Prem Soman, MD, PhD, FRCP (UK), University of Pittsburgh Medical Center-Cardiovascular Institute, A-429 Scaife Hall, 200 Lothrop Street, Pittsburgh, PA 15213; somanp@upmc. edu,premsoman@usa.net.

J Nucl Cardiol 2009;16:173-5.

$1071-3581 / \$ 34.00$

Copyright $(C) 2009$ by the American Society of Nuclear Cardiology. doi:10.1007/s12350-009-9050-8
MPS was read using a standard 17-segment LV model with visual, semi-quantitative scoring for perfusion and regional function. Residual myocardial viability was defined as preserved perfusion (normal to severe hypoperfusion) in $\geq 3$ segments with severe dysfunction (severe hypokinesis, akinesis, or dyskinesis). For comparison with prior studies, this translates into an extent of viable but dysfunctional myocardium involving at least $18 \%$ of the $\mathrm{LV}$ myocardium, and approximates the threshold of critical mass that is generally accepted as clinically significant (i.e., predictive of recovery of function if revascularized, or of an adverse prognosis if not revascularized). ${ }^{1}$ Perfusion assessment however, was based on visual rather than the quantitative (albeit relative) methods previously established as most accurate for detection of viability, and nitrate-enhancement was not performed. ${ }^{2}$ On univariable analysis, patients who died from cardiac causes were older, less often able to undergo exercise testing, and had a greater prevalence of myocardial viability. Among patients who were able to undergo exercise testing, the survivors had greater exercise intensity and duration, with a lower prevalence of combined ischemia and viability, but not ischemia alone. Numerous other clinical and electrocardiographic criteria, and notably all coronary angiographic criteria, were similar between these groups. In a multivariable model that included the entire study group of 167 patients, myocardial viability and the inability to exercise were associated with cardiac death. In patients able to exercise, exercise intensity $<75 \mathrm{~W}$, exercise duration $\leq 5$ minutes (cut-offs determined by ROC curve analysis), myocardial viability and ischemia were associated with cardiac death. The addition of the exercise MPS to the gated MPS variables significantly improved the chi-square of the model, thus establishing incremental prognostic value.

The Candell-Riera study, like any clinical study, has limitations when scrutinized for scientific rigor, and many of these are acknowledged by the authors. The results however are biologically tenable, and consistent with our understanding of the relationships between exercise capacity, myocardial viability, and outcome in heart failure. It is also one of the few prospectively conducted studies of the prognostic utility of MPS, for 
even the large studies involving several thousands of patients that established the prognostic value of MPS were retrospective analyses. ${ }^{3}$ Therefore, although these results are not unexpected, this study contributes important data to an otherwise sparsely populated field.

Prognosis in heart failure is intricately related to etiology. We know from epidemiological and clinical trial data that $60-70 \%$ of patients with heart failure have coronary artery disease (CAD), and fare worse than patients with heart failure which is unrelated to CAD. ${ }^{4}$ However, it must be emphasized that the mere presence of CAD in patients with heart failure implies neither a causal relationship nor adverse prognosis. This was elegantly demonstrated in a study by Felker et $\mathrm{al}^{5}$ of heart failure patients in the Duke database. When these patients were followed up, it became clear that patients with LV systolic dysfunction and single-vessel CAD have a prognosis similar to patients with LV dysfunction and no CAD, which was distinctly better than patients with $\mathrm{LV}$ dysfunction and extensive CAD (defined in that study as at least two-vessels with $>70 \%$ stenosis, single vessel disease of the left main stem or proximal LAD, prior myocardial infarction, or coronary revascularization). This prognostic distinction between patients with ischemic LV dysfunction and patients with limited CAD co-existing with nonischemic LV dysfunction is important, since recent studies seem to indicate that the performance characteristics of MPS are excellent for the detection of extensive CAD (as defined in the Felker study) in new-onset heart failure patients, but only modest for limited CAD. ${ }^{6}$ The Candell-Riera study adds further proof to the concept that gated SPECT MPS can reliably detect prognostically significant $\mathrm{CAD}$ in patients with heart failure. The study fell short of demonstrating a significant difference in outcome between patients who were treated medically and those who underwent revascularization. It was probably underpowered to do so, and not randomized to treatment strategy. The literature on the interaction of myocardial viability, treatment strategy, and patient outcome is limited by a conspicuous lack of randomized clinical trials. However, a meta-analysis of several observational datasets suggested that compared to medical therapy alone, coronary revascularization affords a survival benefit to heart failure patients with significant amounts of viable myocardium, but not to patients with predominantly non-viable myocardium. ${ }^{7}$ The mechanistic basis of this relationship was demonstrated in a study by Senior et $\mathrm{al}^{8}$ who showed that revascularization of viable myocardium results in an improvement in the sphericity index of the left ventricle, indicative of reverse remodeling, and a decrease in the LV endsystolic volume. These changes were associated with improved survival.
Thus, the composite of currently available data in the literature suggests that heart failure patients with extensive myocardial perfusion abnormalities are likely to have etiologically related and prognostically significant $\mathrm{CAD}$ and are therefore likely to benefit from coronary revascularization, while patients with normal MPI are unlikely to have prognostically significant CAD.

One important clinical question which remains is whether MPS can be reliably used in place of coronary angiography to exclude clinically important CAD in patients presenting with new-onset heart failure. While the growing evidence base increasingly suggests that this might be the case, a large prospective trial is needed to answer this question definitively. An important concern is that of balanced ischemia which is particularly relevant to the heart failure population. While the prevalence of balanced ischemia is likely to be low in heart failure patients without angina, stress induced ECG changes or perfusion abnormalities, this has not yet been demonstrated conclusively in a prospective clinical study. Therefore, given the critical importance of excluding significant CAD in this population, patients with new-onset heart failure continue to undergo coronary angiography for the exclusion of CAD.

MPS has also been applied to heart failure patients for indications beyond the assessment of CAD. A particularly promising use is for the assessment of LV dyssynchrony using phase analysis. ${ }^{9}$ Preliminary studies suggest that dyssynchrony parameters derived from gated MPS may be useful to predict response to cardiac resynchronization therapy. ${ }^{10}$ The high reproducibility of this completely automated application is likely to be a significant advantage over echocardiography, which is currently the most widely used imaging modality for this purpose, but limited by poor reproducibility. ${ }^{11}$

Another potentially useful application of gated MPS in heart failure is for the derivation of the LV shape index (the ratio of the short and long axis dimensions of the LV) analogous to the sphericity index derived from echocardiography, which is a measure of the degree of LV remodeling. ${ }^{12}$ Extrapolating from the data on sphericity index, the LV shape index may predict prognosis, and functional response to interventions such as coronary revascularization and CRT. In preliminary studies, the LV shape index has been shown to correlate with symptoms and hospitalization for congestive heart failure, ${ }^{13}$ and with response to beta blocker therapy. ${ }^{14}$

Thus, the many facets of information derived from gated-SPECT MPS including rest and stress myocardial perfusion, viability information, LV volumes, shape indices, and phase analysis can all be applied with advantage to the evaluation of patients with heart failure. In this environment of burgeoning and competing 
new imaging modalities, these developments in the field of MPS are encouraging. For, as recent times have shown us, it is robust science and supporting data that drive the utilization and reimbursement of medical technology. The continuing evolution of supporting data for the use of MPS ensures that its future is bright, even scintillating!

\section{References}

1. Ragosta M, Beller GA, Watson DD, Kaul S, Gimple LW. Quantitative planar rest-redistribution $201 \mathrm{Tl}$ imaging in detection of myocardial viability and prediction of improvement in left ventricular function after coronary bypass surgery in patients with severely depressed left ventricular function. Circulation 1993;87:1630-41.

2. Bisi G, Sciagra R, Santoro GM, Fazzini PF. Rest technetium-99m sestamibi tomography in combination with short-term administration of nitrates: Feasibility and reliability for prediction of postrevascularization outcome of asynergic territories. J Am Coll Cardiol 1994;24:1282-9.

3. Klocke FJ, Baird MG, Lorell BH, Bateman TM, Messer JV, Berman DS, et al. ACC/AHA/ASNC guidelines for the clinical use of cardiac radionuclide imaging-executive summary: A report of the American College of Cardiology/American Heart Association Task Force on Practice Guidelines (ACC/AHA/ASNC Committee to Revise the 1995 guidelines for the clinical use of cardiac radionuclide imaging). Circulation 2003;108:1404-18.

4. Gheorghiade M, Sopko G, De Luca L, Velazquez EJ, Parker JD, Binkley PF, et al. Navigating the crossroads of coronary artery disease and heart failure. Circulation 2006;114:1202-13.

5. Felker GM, Shaw LK, O'Connor CM. A standardized definition of ischemic cardiomyopathy for use in clinical research. J Am Coll Cardiol 2002;39:210-8.
6. Soman P, Lahiri A, Mieres JH, Calnon DA, Wolinsky D, Beller GA, Sias T, Burnham KM, Conway L, McCullough P, Daher E, Walsh MN, Wight J, Heller GV, Udelson JE. Etiology and pathophysiology of new-onset heart failure: Evaluation by myocardial perfusion imaging. J Nucl Cardiol 2009;16:82-91.

7. Allman KC, Shaw LJ, Hachamovitch R, Udelson JE. Myocardial viability testing and impact of revascularization on prognosis in patients with coronary artery disease and left ventricular dysfunction: A meta-analysis. J Am Coll Cardiol 2002;39:1151-8.

8. Senior R, Lahiri A, Kaul S. Effect of revascularization on left ventricular remodeling in patients with heart failure from severe chronic ischemic left ventricular dysfunction. Am J Cardiol 2001;88:624-9.

9. Chen J, Henneman MM, Trimble MA, Bax JJ, Borges-Neto S, Iskandrian $\mathrm{AE}$, et al. Assessment of left ventricular mechanical dyssynchrony by phase analysis of ECG-gated SPECT myocardial perfusion imaging. J Nucl Cardiol 2008;15:127-36.

10. Henneman MM, Chen J, Dibbets-Schneider P, Stokkel MP, Bleeker GB, Ypenburg C, et al. Can LV dyssynchrony as assessed with phase analysis on gated myocardial perfusion SPECT predict response to CRT? J Nucl Med 2007;48:1104-11.

11. Chung ES, Leon AR, Tavazzi L, Sun JP, Nihoyannopoulos P, Merlino J, et al. Results of the predictors of response to CRT (PROSPECT) trial. Circulation 2008;117:2608-16.

12. Douglas PS, Morrow R, Ioli A, Reichek N. Left ventricular shape, afterload and survival in idiopathic dilated cardiomyopathy. J Am Coll Cardiol 1989;13:311-5.

13. Abidov A, Slomka PJ, Nishina H, Hayes SW, Kang X, Yoda S, et al. Left ventricular shape index assessed by gated stress myocardial perfusion SPECT: Initial description of a new variable. $\mathrm{J}$ Nucl Cardiol 2006;13:652-9.

14. Fukuchi K, Yasumura Y, Kiso K, Hayashida K, Miyatake K, Ishida Y. Gated myocardial SPECT to predict response to betablocker therapy in patients with idiopathic dilated cardiomyopathy. J Nucl Med 2004;45:527-31. 